\title{
Anerkennung und ontologische (Un-)Sicherheit von migrantischen Care-Arbeiterinnen in Singapur: Zur Bedeutung von Sichtbarkeit und Zugehörigkeit
}

\author{
Janina Dobrusskin and Ilse Helbrecht \\ Humboldt-Universität zu Berlin, Berlin, Deutschland \\ Correspondence: Janina Dobrusskin (janina.dobrusskin@hu-berlin.de) and Ilse Helbrecht \\ (ilse.helbrecht@geo.hu-berlin.de)
}

Received: 1 April 2021 - Revised: 20 September 2021 - Accepted: 8 October 2021 - Published: 17 November 2021

Kurzfassung. Migrant domestic workers in Singapore are situated in a highly hierarchical environment, requiring them to live and work in the same place. This spatial situation leads to a lack of space and time for the female workers which challenges their psychosocial well-being. We ask how the women, nevertheless, develop psychosocial well-being, based on their recognition and subjective embodied positioning, analytically grasped through the concept of ontological (in)security. Based on qualitative interviews, we show how the workers perceive and produce ontological (in)security through spatial dimensions of visibility and belonging. These dimensions are subjectively embodied in multiple places and networks. On behalf of multiple visibilities and belongings outside the domestic realm, domestic care workers in Singapore are able to actively produce ontological security and strengthen their psychosocial well-being. The results show the relevance of implementing regulations for the women to have more possibilities in choosing their whereabouts.

\section{Einleitung}

Care-Arbeit wird überwiegend von Frauen ${ }^{1}$ geleistet. Sie umfasst meist unbezahlte Haus- und Sorgearbeit wie Putzen, Kochen, Einkaufen, Kinderbetreuung und Pflege älterer Menschen. An vielen Orten der Welt übernehmen zunehmend Migrantinnen aus weniger wohlhabenden Ländern diese Tätigkeiten, was auf einen erhöhten Bedarf an Sorgearbeit durch Alterung und eine größere Teilhabe von Frauen auf dem Arbeitsmarkt - ohne eine entsprechende Übernahme von Sorgearbeit durch Männer - zurückzuführen ist (Summerfield et al., 2006). Migrantische Care-Arbeit basiert auf strukturellen Ungleichheiten und birgt Gefahren für

\footnotetext{
${ }^{1}$ Geschlechtsidentitäten sind biologisch sowie sozial divers und gehen weit über die binären Kategorien von Frau und Mann hinaus. An dieser Stelle finden diese Kategorien dennoch Anwendung, da referierte Statistiken und Studien nur auf dieser Ebene vorliegen. In diesem Artikel wird durchgängig die weibliche Form von „CareArbeiterinnen“ genutzt, da es in unserem Fallbeispiel Singapur ausschließlich Frauen erlaubt ist, in der migrantischen Care-Arbeit tätig zu sein.
}

das Wohlergehen der Frauen (Lan, 2003a; Fong und Yeoh, 2020). Nach Angaben der Internationalen Arbeitsorganisation (ILO) gibt es weltweit etwa 11,5 Millionen migrantische Care-Arbeiterinnen (ILO, 2015). Besonders präsent ist das Phänomen in Ost- und Südostasien, wo etwa ein Drittel der weltweiten Care-Arbeiterinnen tätig sind (Fong und Yeoh, 2020). Hier stellen die außergewöhnliche Arbeitsumgebung der Frauen und die damit zusammenhängenden Konsequenzen für ihr soziales, psychologisches und ökonomisches Wohlergehen ein akutes Forschungsdesiderat dar. Um die Konsequenzen zu verstehen, die sich aus den Arbeitsbedingungen der Care-Arbeiterinnen ergeben, bedarf es insbesondere Untersuchungen zu der besonderen räumlichen Konstellation, in der sie leben und arbeiten (ebd.:704).

Diesen Zusammenhang analysieren wir im Folgenden am Beispiel von Singapur. Im Jahr 2019 war hier bereits in jedem fünften Haushalt eine Care-Arbeiterin angestellt (MOM, 2021a). Die Gesetzgebung bezüglich der migrantischen Care-Arbeit in Singapur erlaubt ausschließlich Frauen, als foreign domestic worker tätig zu sein, und verpflichtet sie, im selben Haushalt zu leben, in dem sie arbeiten. $\mathrm{Zu}$ - 
sammen mit der Bedingung, dass der Aufenthalt der Frauen an ihr Arbeitsverhältnis gebunden ist, sowie vor dem Hintergrund sehr beschränkter Arbeitsrechte führt diese Verpflichtung zu prekären Lebenssituationen (Huang und Yeoh, 2007; Marti, 2019; Parreñas et al., 2020). Den Zusammenhang zwischen der räumlichen Konstellation und dem psychosozialen Wohlergehen der Frauen untersuchen wir im Folgenden anhand des Konzepts der ontologischen (Un-)Sicherheit, welches das subjektive Vertrauen in die Selbstidentität und die eigene Positionierung in der Welt beleuchtet. Es hat in den letzten Jahren zunehmende Verwendung gefunden, so auch in unserem Forschungsfeld der emotionalen und feministischen Geographien (Bondi, 2014; Botterill et al., 2019, 2020; Hopkins et al., 2019). Neben einem oft rezipierten zeitlichen Zugang liegt ein bisher unterbelichtetes Potenzial des Konzepts in der Betrachtung der räumlichen Dimensionen bei der Ver(un)sicherung des Selbst. Die Situation der Care-Arbeiterinnen in Singapur dient dem vorliegenden Beitrag als Beispiel, um die räumliche Herstellung ontologischer (Un-)Sicherheit zu untersuchen. Wir fragen also danach, wie Care-Arbeiterinnen trotz prekärer Arbeits- und Lebensbedingungen ihr psychosoziales Wohlergehen stärken und über räumliche Strategien und Praktiken eine (ontologische) Versicherung des Selbst erlangen.

$\mathrm{Zu}$ diesem Zweck legt der Beitrag zunächst dar, in welche strukturellen Ungleichheiten Care-Arbeiterinnen über die Kategorien gender, class und race eingebettet sind und wie sie darüber in ihren Arbeitsverhältnissen Ausbeutung und Unterdrückung erfahren. Anschließend nehmen wir die Arbeitsumgebung der Care-Arbeiterinnen zum Ausgangspunkt, um ihr Wohlergehen zu betrachten. Studien zum Wohlergehen von Care-Arbeiterinnen berücksichtigen räumliche Dimensionen bisher vorrangig über die Unterscheidung von privaten und öffentlichen Räumen sowie über sozialräumliche Grenzziehungen innerhalb der Arbeitsverhältnisse. Weitere sozialräumliche Dimensionen, die Einfluss auf das Wohlergehen der Frauen haben und damit Stellschrauben für Verbesserungen sein könnten, bleiben bisher unterbelichtet. Basierend auf der Analyse von 60 narrativen Interviews mit der Methode der Foto-Elizitation erläutern wir, inwiefern sich die Positionierung und Anerkennung der CareArbeiterinnen in dem Erleben von Sichtbarkeit und Zugehörigkeit abbildet und dadurch das psychosoziale Wohlergehen der Frauen beeinflusst. ${ }^{2}$ Innerhalb des Haushalts, in dem sie arbeiten, als Person und in ihrer Tätigkeit nicht gesehen zu werden oder gezeigt zu bekommen, dass sie als Familienmitglied nicht anerkannt werden, führt zu Verunsicherung. Um ihre ontologische Sicherheit zu stärken, suchen CareArbeiterinnen Sichtbarkeit und Zugehörigkeit auf multiplen Ebenen - in unterschiedlichen Räumen und Netzwerken. Die Ergebnisse der Studie weisen auf die Notwendigkeit hin, mi-

\footnotetext{
${ }^{2}$ Die Untersuchung wurde gefördert von der Deutschen Forschungsgemeinschaft (DFG) im Rahmen des Sonderforschungsbereiches 1265 „Re-Figuration von Räumen“.
}

grantischen Care-Arbeiterinnen strukturell Zugang zu vielfältigen Räumen und Gemeinschaften außerhalb ihrer Arbeitsräume zu ermöglichen.

\section{Strukturelle Ungleichheit und Vulnerabilität in der Care-Arbeit}

Die Anzahl weltweit tätiger Migrantinnen in der Care-Arbeit hat in den letzten Jahrzehnten stetig zugenommen - wie auch das wissenschaftliche Interesse an ihrer Tätigkeit (Ogawa et al., 2018; Fong und Yeoh, 2020; Aulenbacher et al., 2021). Migrantische Care-Arbeit ist ein Phänomen globaler Arbeitsteilung vor dem Hintergrund kapitalistischer Märkte. Die Frauen erleben dabei eine hohe Vulnerabilität durch prekäre Arbeitsbedingungen sowie durch strukturelle Ungleichheiten. Pei-Chia Lan hebt gender, class und race als dominant wirkende Dimensionen der Ungleichheit migrantischer CareArbeit hervor (Lan, 2003a:187). Mit dem Begriff der feminization von Arbeitsmigration haben Brenda Yeoh et al. bereits im Jahr 2005 darauf verwiesen, dass Frauen deshalb immer öfter eine Arbeit an anderen Orten der Welt aufnehmen, um ihre Familien zu Hause zu unterstützen. Neben Singapur und den Golfstaaten sind insbesondere Nordamerika sowie Südund Westeuropa Aufnahmeregionen von Care-Arbeiterinnen aus weniger wohlhabenden Ländern (Aulenbacher et al., 2021:8). Lan verhandelt die Identität der Frauen unter den Kategorien „maid or madam“ (Lan, 2003a), zwischen denen sie wechseln bzw. die auch gleichzeitig relevant sein können: So sind die Frauen gegenüber der eigenen Familie madam, während sie in ihrem Arbeitsverhältnis zur maid werden. Unter Rückgriff auf eine transnationale Perspektive wird zudem zwischen den multiplen Class-Identitäten der Frauen differenziert, die in verschiedenen Räumen unterschiedliche Positionen einnehmen (Rother, 2017:969): So werden Care-Arbeiterinnen auf den Philippinen und in Indonesien als nationale Heldinnen gefeiert, ${ }^{3}$ wohingegen ihnen am Ort ihrer Migration eine Zugehörigkeit zur Klasse der Hausangestellten zugeschrieben wird. Über ihr Bewusstsein für den transnationalen Charakter der individuell erlebten Ausbeutung nehmen sich die Arbeiterinnen schließlich als Teil einer transnationalen sozialen Klasse wahr, was sie zu ihrer politischen Organisierung ermächtigt. Über das Annehmen der unterschiedlichen Identitäten erlangen Care-Arbeiterinnen eine Form von Handlungsmacht (ebd.). Schließlich adressiert Pei-Chia Lan über die favorisierte Anstellung von Migrantinnen im niedrig bezahlten Care-Sektor die dritte Dimension der Ungleichheit, welche zu einer ethnisch-stratifizierten Arbeitsteilung führt, die schließlich auch rassistische Diskurse befördert (Lan, 2003b).

Die dargestellten strukturellen Ungleichheiten innerhalb der Care-Arbeit entlang der Dimensionen von gender, class

\footnotetext{
${ }^{3}$ Die Bewertung der Arbeitsmigration ist nicht nur positiv - so wird den Frauen zum Teil auch vorgeworfen, ihre eigenen Familien zu vernachlässigen (Pratt und Johnston, 2014).
} 
und race sind keinesfalls folgenlos. Care-Arbeiterinnen sind einem breiten Spektrum an Vulnerabilitäten ausgesetzt, die ihr soziales, physisches und psychologisches Wohlbefinden beeinflussen (Fong und Yeoh, 2020:704):

"It has a clear structure of exploitation, as migrant domestic workers usually work long hours with unclear guidelines. As migrant workers, these domestic workers have little say when employers control the standards of their working environment, sometimes even revoking visas without notice." (Ebd.:522)

Neben den strukturellen Ungleichheiten, aufgrund derer Arbeitgeber:innen prekäre Arbeitsbedingungen durchsetzen können, haben auch die geltenden Gesetzgebungen sowie die sich daraus ableitende räumliche Konstellation des Arbeitsumfeldes Einfluss auf das Wohlergehen der Frauen (ebd.; Yeoh und Huang, 2010). Die besondere räumliche Situation von sogenannten Live-in-Arrangements bedeutet, dass der Arbeitsplatz der Frauen gleichzeitig das Zuhause ihrer Arbeitgeber:innen ist und oftmals - wie im Fall von Singapur - auch verpflichtend das von ihnen selbst (Lai und Fong, 2020).

Statistische Untersuchungen belegen, dass hierbei ein direkter Zusammenhang zwischen beengten räumlichen Bedingungen einerseits und Erfahrungen missbräuchlichen Verhaltens durch Arbeitgeber:innen andererseits besteht (ebd.; Yu, 2016). So zeigt eine Studie in Hong Kong, dass die Wohnungsgröße einen Einfluss darauf hat, wie wahrscheinlich es ist, Aggressionen der Arbeitgeber:innen ausgesetzt zu sein. Demnach werden Care-Arbeiterinnen, die in kleineren Wohnungen arbeiten, häufiger von ihren Arbeitgeber:innen gescholten (Lai und Fong, 2020:732).

Demgegenüber haben Care-Arbeiterinnen mit einem eigenen Zimmer seltener das Gefühl, von ihren Arbeitgeber:innen vernachlässigt zu werden, da Bedürfnisse von ihnen anerkannt werden (ebd.). Eine Studie in Singapur stellte fest, dass rund 40 Prozent von 472 befragten CareArbeiterinnen über kein eigenes Zimmer verfügen und sich entgegen den Vorgaben teilweise auch Zimmer mit männlichen Jugendlichen oder Erwachsenen teilen (Yu, 2016). Wie diese Studien zeigen, trägt die räumliche Konstellation des Live-in-Arrangements zur Vulnerabilität von CareArbeiterinnen bei. Die dargelegten räumlichen Bedingungen beeinflussen das psychosoziale Wohlergehen der Frauen. Ihre Situierung in der spezifischen Arbeitsumgebung geht jedoch über die rein materielle Anordnung hinaus und basiert auch auf räumlichen Praktiken, Vorstellungen und sozialen Strukturen. Wie migrantische Care-Arbeiterinnen psychosoziales Wohlergehen über ihre sozialräumliche Positionierung erleben, wahrnehmen und schließlich auch aktiv herstellen, betrachten wir im Folgenden.

\section{Psychosoziales Wohlergehen migrantischer Care-Arbeiterinnen}

Studien zum Wohlergehen migrantischer Care-Arbeiterinnen nehmen vor allem die ausbeuterischen und unterdrückenden Arbeitsbedingungen zum Ausgangspunkt (Huang und Yeoh, 2007; Fong und Shibuya, 2020) und fordern gute Arbeitsbedingungen ein (Aulenbacher et al., 2021). Die besondere sozialräumliche Konstellation des Arbeitsverhältnisses spielt in diesen Studien eine immanente, aber hintergründige Rolle. Bereits in "Maid to order in Hong Kong“ (1997), einem Pionierwerk der transnationalen Migrationsstudien, das die Machtdynamiken und die Formen der (Selbst-)Disziplinierung und Überwachung innerhalb der Arbeitsverhältnisse in den Vordergrund stellt, argumentiert Nicole Constable:

"[T]he confluence of home and workplace makes it more difficult for household workers than factory workers to separate work spatially, it is also more difficult temporally to distinguish time on and off." (Constable, 1997:89)

Den besonderen sozialräumlichen Herausforderungen des Arbeitsverhältnisses begegnen die Frauen mit der Notwendigkeit sozialräumlicher Grenzziehungen, die Pei-Chia Lan (2003b) über die Sphären des Privaten und des Öffentlichen erläutert. Ihre Studie zeigt, wie Arbeitgeber:innen den Einschluss der Care-Arbeiterinnen in das Private der Familie bzw. ihren Ausschluss davon aktiv suchen und wie zum anderen Care-Arbeiterinnen in Ermangelung von Privatsphäre öffentliche Orte an ihrem Wohnort nutzen (ebd.). Es wird deutlich, wie die Frauen vor dem Hintergrund der herausfordernden räumlichen Konstellation und in teilweise sehr prekären Arbeitssituationen Strategien für ihr Wohlergehen entwickeln und sich ihrer Selbst in dem spezifischen Arbeitskontext vergewissern.

Das Wohlergehen und die Sicherheit von migrantischen Care-Arbeiterinnen wird jüngst zunehmend mittels quantitativer Studien untersucht (Chiu, 2018; Wang und Liu, 2018; van Bortel et al., 2019; Chung et al., 2020). Einige haben etwa herausgearbeitet, dass es den Frauen an Raum und Zeit fehlt, um Stress zu bewältigen und darüber ihr Wohlergehen zu verbessern (Wang und Liu, 2018:132; van Bortel et al., 2019:10). Auf einer räumlichen Ebene wird daher gefordert, das Arbeitsumfeld der Care-Arbeiterinnen neu zu gestalten und beispielsweise unterschiedliche Wohnmodelle zu ermöglichen, sodass die Frauen alternativ auch außerhalb des Haushalts ihrer Arbeitgeber:innen wohnen können. Die Forderung ist also, neben Anstellungsverhältnissen in einem „home-based living“ ein Modell des ,out-of-home living“ zu entwickeln (Wang und Liu, 2018:134). Neben solchen politischen Handlungsempfehlungen werden psychosoziale Interventionen geltend gemacht, die das Wohlergehen der Migrantinnen in der Care-Arbeit verbessern sollen (Hall et al., 
2019). Wie entsprechende Interventionen ausgestaltet sein können, bleibt jedoch offen.

In diesem Beitrag erläutern wir, wie psychosoziales Wohlergehen mit räumlichen Dimensionen zusammenhängt und wie darauf aufbauende Interventionen aussehen können. Hierfür ziehen wir das Konzept der ontologischen (Un-)Sicherheit heran. Es geht zurück auf den Psychiater Ronald D. Laing und wurde für die Sozialwissenschaften von Anthony Giddens weiterentwickelt. Er beschreibt ontologische Sicherheit wie folgt:

"The phrase refers to the confidence that most human beings have in the continuity of their selfidentity and in the constancy of the surrounding social and material environments of action." (Giddens, 1990:92)

Ontologische Sicherheit ist demnach eine Grundvoraussetzung für psychische Gesundheit und Wohlbefinden (Giddens, 1991:38f.; Vaquera et al., 2017). Sie ist charakterisiert durch zweierlei: erstens eine subjektiv empfundene Gewissheit über die Kontinuität des Selbst und die eigene Identität und zweitens das Vertrauen in die Verlässlichkeit der sozialen und materiellen Umgebung und damit in die eigene Positionierung in der Welt. Somit ist die Räumlichkeit ein wesentlicher Bestandteil subjektiver ontologischer Ver(un)sicherungen. Gerade der Zusammenhang zwischen dem subjektiven Wohlergehen und der Rolle der sozialen und materiellen Umgebung macht das Konzept der ontologischen Sicherheit für geographische Forschungen ausgesprochen fruchtbar.

Mit unserem Beitrag verfolgen wir konzeptionell zwei Ziele: Zum einen erläutern wir tiefergehend, wie ontologische (Un-)Sicherheit über räumliche Dimensionen hergestellt wird. Dabei zeigen wir empirisch, dass insbesondere die (Un-)Sichtbarkeit der Care-Arbeiterinnen ein entscheidender Faktor für ihre soziale Anerkennung bzw. NichtAnerkennung - und damit auch wesentlicher Faktor ihrer ontologischen Ver(un)sicherung ist. Das Konzept der Anerkennung erweist sich für die Untersuchung der räumlichen Dimensionen von ontologischer Ver(un)sicherung besonders hilfreich, denn es berührt - ähnlich wie der Begriff der ontologischen Sicherheit - existenzielle Erfahrungen des Menschen und Fragen der Subjektwerdung (Honneth, 2003:91). Mit Axel Honneth (ebd.:26f.) verstehen wir dabei unter „Anerkennung, die bewertende Wahrnehmung von Personen, die oftmals auf expressiven Gesten anderen gegenüber beruht und die „Bekundung einer Wertschätzung“ umschließt (ebd.:27). Dabei ist prinzipiell davon auszugehen, dass eine solche ,gegenseitige Anerkennung ständig umkämpft ist“" (Gadamer, 1990:365). Zum anderen erweitern wir mit unserer Studie die Literatur zum Wohlbefinden der Care-Arbeiterinnen um eine subjektiv verkörperte Perspektive, die sowohl soziale als auch psychische Handlungsbezüge berücksichtigt. Dem zentralen Interesse einer feministischen Geopolitik folgend räumen wir dabei Maßstäben,
Räumen, Subjekten und Praktiken eine politische Relevanz ein, die in dominanten geopolitischen Diskursen marginalisiert werden (Hörschelmann und Reich, 2017:74; Hyndman, 2004; Massaro und Williams, 2013). Auch schließen wir an eine psychoanalytische Perspektive innerhalb der Geographie an, die genutzt werden kann, um über persönliche und subjektive emotionale Geographien nachzudenken (Bondi, 2014:345). Unter Rückgriff auf das Konzept der ontologischen (Un-)Sicherheit - und unter Einbezug der kritischen Theorie der Anerkennung - analysieren wir das Vertrauen der Care-Arbeiterinnen bezüglich ihrer Positionierung in der Welt. Diese Perspektive erlaubt es uns zu zeigen, wie Care-Arbeiterinnen in Singapur ein Vertrauen in die eigene Positionierung auf einer affektiven, emotionalen und subjektiv verkörperten Ebene herstellen. Liz Bondi verweist darauf, dass es sich bei der Frage nach dem Empfinden von Unsicherheit nicht um einen Ausnahmezustand handelt: ,[E]lements of ontological insecurity are an ordinary part of all our lives, rendering fragile our sense of being in the world" (ebd.). Das Maß, in dem sich eine Person ontologisch (un-)sicher fühlt, ist einem stetigen Aushandlungsprozess unterworfen. Wir nutzen daher die Schreibweise der „ontologischen (Un-)Sicherheit“, um auf die Veränderlichkeit des Empfindens und seine Dialektik hinzuweisen.

\section{Fallbeispiel Singapur und methodisches Vorgehen}

Von 2015 bis 2020 stieg die Anzahl der in Singapur als foreign domestic worker tätigen Frauen von 231.500 auf 252.600 an (MOM, 2021a). Damit macht der Anteil der Care-Arbeiterinnen knapp 19 Prozent aller ausländischen Arbeiter:innen in Singapur aus (ebd.). Voraussetzungen für die Anstellung als Care-Arbeiterin in Singapur sind, dass die Frauen zum Zeitpunkt der Bewerbung zwischen 23 und 50 Jahre alt sind, mindestens acht Jahre einer formalen Bildung nachgegangen sind und aus einem der durch das Arbeitsministerium anerkannten Herkunftsländer stammen (MOM, 2021b). Als Herkunftsländer der Care-Arbeiterinnen am relevantesten sind Indonesien $(50 \%)$, die Philippinen (30\%) und Myanmar (15\%), wobei entsprechende Daten auf Hochrechnungen beruhen (Yu, 2016). Daten zu den Arbeitsbedingungen der Care-Arbeiterinnen vom Arbeitsministerium Singapurs (MOM, 2016) erscheinen nicht sonderlich belastbar. Diese weichen stark von denen migrantischer Arbeitnehmerinnen-Organisationen wie HOME (Humanitarian Organization for Migration Economics) (Wessels, 2015) und TWC2 (Transient Workers Count Too) (Yu, 2016) ab. Auch eine neuere Studie der Internationalen Arbeitsorganisation in Zusammenarbeit mit der UN (ILO, 2019) kommt zu anderen Ergebnissen. Generell gilt, dass Singapur keinen Mindestlohn für Care-Arbeit vorgibt, das jeweilige Herkunftsland jedoch Konditionen festgelegen kann (Marti, 2019). Als durchschnittliches Monatsgehalt wird 650 Singapur-Dollar (SGD) angegeben (ILO, 2019:36). Ar- 
beitserfahrung kann zu einem höheren Einkommen führen, wohingegen Überstunden generell nicht vergütet werden. Außerdem sind Care-Arbeiterinnen oft verschuldet, da sie den Agenturen, die sie aus ihren Herkunftsländern nach Singapur vermittelt haben, Gebühren zahlen müssen (Wessels, 2015:69). Die Arbeitszeit der Frauen variiert recht stark, wobei der Durchschnitt bei 13 Arbeitsstunden (ebd.:52) bis 14,5 Arbeitsstunden (Yu, 2016:17) am Tag liegt. Misshandlungen durch Arbeitgeber:innen finden auf unterschiedlichen Ebenen statt: ökonomisch $(35 \%)$, verbal $(51 \%)$, physisch (6\%), moralisch (7\%) und sexuell (7\%) (Wessels, 2015:37). Auf die Frage, ob die Frauen von ihren Arbeitgeber:innen menschlich behandelt werden, antworteten 73 Prozent mit „oft“ oder ,immer“, die anderen 27 Prozent antworten mit „manchmal“, „selten“ oder „,nie“ (ebd.:42). Die gesetzliche Verpflichtung, dass der Arbeitsort der Care-Arbeiterinnen gleichzeitig ihr Wohnraum ist, sowie die Regelung, dass ihr Aufenthalt an einen Arbeitsvertrag geknüpft ist, machen es den Frauen besonders schwer, sich dieser Situation zu entziehen. Zudem ist die Fläche des Inselstaates natürlich begrenzt und dicht besiedelt, sodass Wohnraum knapp ist (Mele, 2017). Diese Raumknappheit zusammen mit dem geringen Wert, der den Care-Arbeiterinnen offenbar beigemessen wird, führen dazu, dass sie oft über kein eigenes Zimmer verfügen: 40 Prozent der Frauen teilen sich ihren Schlafraum mit anderen Haushaltsmitgliedern (Yu, 2016). Dadurch wird eine Abgrenzung zwischen Arbeitsplatz und Wohnraum praktisch unmöglich und die Wahrscheinlichkeit von Missbrauch und Misshandlung steigt (ebd.). Wie diese Daten zeigen, stellen sowohl die Arbeitsbedingungen als auch die räumliche Situation der Care-Arbeiterinnen in Singapur eine Herausforderung für ihr Wohlbefinden dar.

Unsere Studie basiert auf 60 qualitativen Interviews mit einem divers zusammengesetzten Sample der Bevölkerung Singapurs (in Bezug auf Gender, Alter, sozioökonomischer und ethnischer Herkunft). Diese wurden mit der Methode der Foto-Elizitation geführt, wobei von den Forschenden ausgewählte Bilder im Interview als visueller Leitfaden dienen (Dirksmeier, 2015). Der Einsatz der Bilder macht sich die Vielfältigkeit der möglichen Bezüge sowie ihre affektive, emotionale Dimension zunutze, ,um den interviewten Personen Aspekte zu entlocken, die bei rein kognitivsprachlichen Impulsen oftmals latent bleiben“ (Dobrusskin et al., 2021:212). Die Auswahl der von uns als Gesprächsimpuls eingesetzten Fotos bedient verschiedene Maßstabsebenen, Raumtypen und unterschiedliche Markierungen von (Un-)Sicherheit. Darüber hinaus wurde die Auswahl davon geleitet, den Gesprächspartner:innen einen möglichst großen Interpretationsspielraum zu geben (ebd.). Die eingesetzten Bilder zeigen beispielsweise eine improvisierte Schlafstätte in einem Innenraum, eine Kunstinstallation an einem Grenzzaun oder eine Viehherde mit Hirten vor der Teilansicht einer Stadt. Es wurden stets die gleichen fünf Fotos gezeigt und im Anschluss wurde ein Set von weiteren acht Bildern zur freien Auswahl gereicht. In der Gesprächsführung wurde der
Bezug zum Erlebten, zum Alltag und zu den Emotionen der Gesprächspartner:innen gesucht, um die alltägliche Herstellung ontologischer (Un-)Sicherheit über räumliche Bezüge zu betrachten.

Durch den offenen Ansatz des Forschungsprojekts wurden unterschiedliche Themen adressiert, wie beispielsweise die sicherheitsstiftende Funktion des Zuhauses oder existenzielle Bedrohungen, die von Prozessen der Globalisierung ausgehen (Pohl et al., 2020; Helbrecht et al., 2021). Besonders häufig wurde die räumliche Beengtheit des Wohnens in Singapur (oft thematisiert durch das Bild der improvisierten Schlafstätte) und die Relevanz von Sorgebeziehungen hervorgehoben (beispielsweise ausgelöst durch das Bild der Kunstinstallation, die ein Kleinkind abbildet, das über den Grenzzaun greift). Da die räumliche Konstellation der Arbeits- und Lebenssituation für Care-Arbeiterinnen auffallend prekäre Auswirkungen hat, spitzt sich in ihrer Situation die Frage nach der Herstellung ontologischer Sicherheit besonders zu. Neben Interviews mit sechs Care-Arbeiterinnen sind die Perspektiven von Arbeitgeber:innen, Mitarbeiter:innen migrantischer Arbeitsorganisationen und anderen Bewohner:innen Singapurs in die Analyse eingeflossen. Der Zugang zu CareArbeiterinnen hat über Multiplikator:innen innerhalb einer migrantischen Arbeitsorganisation stattgefunden, sodass davon ausgegangen werden kann, dass eher privilegierte CareArbeiterinnen an den Interviews teilgenommen haben. Dadurch, dass eine weiße Forscherin aus Europa die Interviews in englischer Sprache führte, waren diese teilweise sprachlich limitiert und ist der Interviewsituation ein Hierarchiegefälle eingeschrieben. Die Positionalität der Forscherin, die vor Ort die Rolle einer (privilegierten) Außenseiterin einnimmt, konnte in einigen Gesprächen zu einer besonders großen Erzählbereitschaft führen - beispielsweise gegenüber Personen, die selbst eine Außenseiter:innenrolle einnehmen. In anderen Gesprächen fiel auf, dass das gegenseitige Verstehen durch fehlendes lokales Wissen sowie sprachliche Limitierungen nur begrenzt möglich war. Die Analyse der Interviews fand über einen induktiven Kodierungsprozess mit MAXQDA und eine inhaltsanalytische Interpretation der Interviewtranskripte statt, wobei die Relevanz der Kategorien und räumlichen Dimensionen der Sichtbarkeit und Zugehörigkeit in ihren vielfältigen Bezügen zum psychosozialen Wohlergehen deutlich zum Tragen kam.

\section{Ergebnisse und Diskussion}

Mit den Ergebnissen der Analyse zeigen wir im Folgenden, wie Care-Arbeiterinnen zur Stärkung ihrer ontologischen Sicherheit agieren und welche sozialräumlichen Strategien sie dafür anwenden. Insbesondere über die Dimensionen der Sichtbarkeit und der Zugehörigkeit positionieren sich die Frauen aktiv und stellen darüber ein ontologisches Sicherheitsempfinden her, worauf schließlich ihr subjektiv empfundenes Wohlergehen beruht. Sichtbarkeit ist relatio- 
nal, da über sie eine Beziehung aufgespannt wird - zwischen der Person, die sieht, und der, die gesehen wird (Brighenti, 2010:39). Sichtbarkeit kann ermächtigend und/oder entmachtend wirken, abhängig davon, inwiefern sie Anerkennung mit sich bringt oder als Kontrollinstrument eingesetzt wird (ebd.). Honneth legt in seiner philosophischen Auseinandersetzung zur Unsichtbarkeit dar, dass eine Person physisch für andere erst einmal sichtbar sein muss, um sie sozial anzuerkennen oder auch, um ihre Nichtexistenz vorzugeben (Honneth, 2003). Er stellt fest, dass mit Anerkennung stets ,ein existentieller Ruck, ein plötzlicher Wandel im eigenen Selbstverständnis verbunden“ ist (ebd.: 91). Sichtbarkeit über eine intersubjektive Anerkennung kann demnach als konstitutiv für das Personsein verstanden werden und ist ein grundlegendes Element bei der Herstellung ontologischer Sicherheit. Sichtbarkeit im Sinne der sozialen Anerkennung geht der Dimension der Zugehörigkeit - als der Anerkennung des Individuums als Teil einer bestimmten Gemeinschaft voraus.

\subsection{Forderungen nach (Un-)Sichtbarkeit}

Care-Arbeiterinnen in Singapur erleben in ihren Arbeitsverhältnissen regelmäßig, dass Unsichtbarkeit von ihnen verlangt wird. Eine typische Erwartungshaltung an die Frauen ist beispielsweise, dass sie ihr Bett, das sich in einem Gemeinschaftsraum befindet, jeden Morgen wieder abbauen. Emily, die bereits für über ein Dutzend Haushalte als CareArbeiterin tätig war, spricht genervt von dieser Notwendigkeit: „Every morning you have to carry the bed and put it somewhere else." (S37:526) Trotz der Routine, die diese räumliche Praxis mit der Zeit erlangt hat, sitzt die Frustration über diese Notwendigkeit tief. Noch drastischer spitzt sich die Situation zu, wenn sich die Frauen sogar bei der Durchführung ihrer Aufgaben genötigt sehen, die Unsichtbarkeit ihrer Tätigkeiten oder sogar ihrer Person zu wahren. In den Ausführungen von Maria, die seit 19 Jahren als Care-Arbeiterin in Singapur tätig ist, wird dies in der Erzählung über ihre ehemalige Arbeitgeberin deutlich:

"She spoke to me like the husband: 'Oh, Maria, can you please make yourself scarce whenever we are at home.' I thought for revenge. Make myself scarce? How? Since I'm working with you, how would I do that? Make myself scarce. You know, the word scarce hit me. How do they take me with them in the house?" (S05:242f.)

Die Entrüstung in Marias Erzählung ist vor allem von Wut getragen. Die beschriebene Situation bildete den Tropfen, der das Fass zum Überlaufen und Maria schließlich zum Verlassen des Haushalts brachte. Auch Emily berichtet von ihrem aktuellen Anstellungsverhältnis, bei dem das Putzen der Räumlichkeiten im Beisein der Familienangehörigen keine Option sei, ihre Unsichtbarkeit also erwartet werde:

\begin{abstract}
"So, they wake up late. They'll go swimming and like that. So, when they go out for swimming, I have to hurry up to clean everything. Because if they come home, they stay up. When they come back from swimming, they're at home the whole day. So, they drink coffee, drink like this, so I cannot do anything." (S37:600f.)
\end{abstract}

Die Notwendigkeit, Arbeiten auf eine Art und Weise zu erledigen, bei der sie selbst gegenüber den Arbeitgeber:innen nicht sichtbar ist, erzeugt bei Emily Stress und Verunsicherung:

"Because, if I missed one work, I cannot go on so fast. Because, let's say, I missed this. Um, so I become crazy. If I didn't do the things in the right order, I will not be happy." (S37:622f.)

Nur wenn sie die ihr zugewiesenen Aufgaben in einer bestimmten Abfolge erledigt, kann sie die Erwartungshaltung ihrer Arbeitgeber:innen erfüllen, nicht sichtbar zu sein. Wie aus den Erzählungen von Emily und Maria deutlich wird, wird den Frauen ihre Sichtbarkeit aberkannt - an dem Ort, an dem sie arbeiten und leben; an dem Ort, der ihr Zuhause darstellt. Die Forderung nach Unsichtbarkeit während der ihnen zugewiesenen Care-Tätigkeiten ist eine gängige Tendenz. In einem Diskurs zu ,unsichtbarer Arbeit“ wird zudem darauf verwiesen, dass das Verbergen von Arbeit mit einer emotionalen Anstrengung verbunden ist (Crain et al., 2016). Dies wird auch im Fall der Care-Arbeiterinnen in Singapur deutlich.

Unter Rückgriff auf das Konzept der ontologischen (Un-)Sicherheit lässt sich die sicherheitsstiftende Funktion der Sichtbarkeit detaillierter betrachten. Indem von Emily gefordert wird, bei der Ausübung ihrer Care-Tätigkeit nicht in Erscheinung zu treten, wird ihr die Chance auf soziale Anerkennung als Person genommen. Die Situation ist also noch drastischer als die „Demütigung“, die Honneth (2003:12) beschreibt, wenn Menschen ihre soziale Bedeutungslosigkeit kommuniziert wird, indem einer Person durch bewusstes Nicht-zur-Kenntnis-Nehmen gezeigt wird, dass sie sozial nicht anerkannt wird. Im Falle der Care-Arbeiterinnen nehmen die Arbeitgeber:innen nicht einmal diesen Akt des Ignorierens als aktives Nicht-Sehen auf sich, um die Anerkennung zu verweigern. Vielmehr fordern sie sehr viel umfassender die physische (und damit soziale) Unsichtbarkeit der Frauen selbst. Für die Care-Arbeiterinnen bedeutet dies eine starke Verletzung und Verunsicherung ihres Personseins, wie beispielsweise die Frustration von Maria zeigt, die schließlich sogar in die Beendigung des Arbeitsverhältnisses mündet. Über das relationale Verhältnis von Sichtbarkeit definieren sich Subjektpositionen (Brighenti, 2010:39). Nicht gesehen zu werden bedeutet, keine anerkannte Positionierung zugestanden zu bekommen. Es entsteht also eine existenzielle und damit ontologische Verunsicherung durch die Forderung 
nach Unsichtbarkeit, weil die subjektiv empfundene Gewissheit der Care-Arbeiterinnen über ihr materielles Selbst durch die fehlende soziale Anerkennung infrage gestellt wird. Ähnliches beschreibt Zuzana Sekeráková Búriková (2020) für slowakische Care-Arbeiterinnen in London, deren Sichtbarkeit eng verbunden mit sozialer Anerkennung ist und ihnen zu einer Form von Empowerment verhilft.

Entgegen einer Verunsicherung durch die Forderung nach Unsichtbarkeit kann das Erleben von Sichtbarkeit - im Sinne einer Anerkennung des Selbst und seiner Kontinuität - somit schließlich auch ontologische Sicherheit befördern. In den Ausführungen der Care-Arbeiterinnen wird deutlich, dass Sichtbarkeit auf mehreren Ebenen gesucht wird: an verschiedenen Orten und in unterschiedlichen Netzwerken. Auch die zuvor genannte Studie zu slowakischen Care-Arbeiterinnen in London stellt fest, dass die Frauen Sichtbarkeit in ihrem eigenen Zimmer und im öffentlichen Stadtraum suchen (Sekeráková Búriková, 2020). In unserer Studie konnten wir zeigen, wie die Frauen auch innerhalb ihres Arbeitsverhältnisses in spezifischen Situationen Sichtbarkeit erlangen und diese als bestärkend empfinden. So beschreibt Emily in Bezug auf eine Familie, die sie besonders gut behandelt, wie wohl sie sich in ihrer Aufmachung bei einem offiziellen Dinner mit dem Botschaftssekretär bei ihnen zu Hause fühlt:

"I served the foods like in a restaurant in a proper dress. Yeah. I wear a white and black skirt, and small heels. [chuckles] So sexy." (S37:277f.)

Ihr Auftreten zu dem Anlass des hohen Besuchs macht sie stolz, sie erfährt Sichtbarkeit und Anerkennung. Als eine Situation, der sie bei der Erzählung über sich selbst in ihrer Tätigkeit als Care-Arbeiterin Relevanz einräumt, stärkt sie die Gewissheit ihrer Selbst.

Wird Sichtbarkeit in der Gastfamilie nicht in einem befriedigenden Maße erlebt, so wird sie für die Frauen an anderen Orten umso relevanter. Sichtbarkeit ist jedoch nicht direkt mit positiver Anerkennung gleichzusetzen. In der Wahrnehmung der Mehrheitsgesellschaft sind Care-Arbeiterinnen im öffentlichen Raum oft in Gruppen anzutreffen, und diese fallen dann durch störendes Verhalten auf. Wie Honneth feststellt, ist Anerkennung stark an eine Normativität der Wahrnehmung geknüpft - die Entscheidung über das Anerkennen geht damit dem tatsächlichen Erkennen unseres Gegenübers meist voraus (Honneth, 2003:26f.). Vor dem Hintergrund gesellschaftlicher Machtverhältnisse und struktureller Diskriminierung nehmen insbesondere Netzwerke wie diejenigen der institutionalisierten Organisation der Care-Arbeit, aber auch religiöse Gemeinschaften und Freundschaften eine zentrale Rolle für die soziale Anerkennung der Arbeitsmigrantinnen ein. Ihre Möglichkeiten, sich in diesen Netzwerken zu bewegen, sind jedoch durch die hohe Arbeitsbelastung auf spezifische Zeiten und Orte begrenzt. Margarete, die zum ersten Mal in Singapur arbeitet und sich für älter ausgibt, als sie ist, um die Tätigkeit als Care-Arbeiterin ausführen zu dürfen, besucht an ihren freien Sonntagen eine selbst organisierte Akademie der Care-Arbeiterinnen, um sich fortzubilden. Über die Möglichkeit der Begegnung mit Freundinnen berichtet sie:

"I cannot visit them."

Interviewer: "Okay. So, are you meeting here at the academy?"

"Yeah. And once we finish the class, we go to the city hall. City hall is the place where Myanmar people meet, yeah. [...] There is, yeah. [chuckles] All the Myanmar people are there and Myanmar food, yeah. They sell it. We always go there." (S13:195ff.)

Shopping Malls als Enklaven spezifischer Herkunftsgruppen (beispielsweise City Plaza Mall als indonesischer Anlaufpunkt, Lucky Plaza als philippinischer und Peninsula Plaza als myanmarischer) bilden wichtige Orte für die Frauen, um ihren Bedürfnissen nachzugehen, sichtbar zu werden und somit Anerkennung zu erfahren. Ein weiterer Ort, der besonders relevant für Care-Arbeiterinnen ist, ist die Akademie. Hier organisieren sich Arbeitsmigrantinnen, um sich für die Zeit nach der Tätigkeit in Singapur weiterzubilden. Emily, die mittlerweile selbst als Lehrkraft in der Akademie arbeitet, berichtet voller Stolz von ihren Erfolgen in der Zeit ihrer eigenen Ausbildung:

"Then after a year, I'm an honour student. I mean, in the baking and the cosmetology as well. I'm, uh, awarded. I mean, I got the certificate and medal." (S37:410f.)

Neben der Absolvierung von Weiterbildungen mit der Möglichkeit, Zertifikate und Auszeichnungen zu erhalten, können innerhalb der Organisation Aufgaben und institutionalisierte Rollen übernommen werden. Die Verleihung von Abschlüssen findet innerhalb von festlichen Veranstaltungen statt, in denen die Frauen mit ihren unterschiedlichen Positionen Wertschätzung erfahren: Es werden Fotos gemacht, Gäste eingeladen (manchmal auch Arbeitgeber:innen), Medaillen und Zertifikate überreicht und schließlich auch Jahrbücher unterschrieben. Neben diesen Praktiken, die innerhalb der Organisation Sichtbarkeit schaffen, ermöglichen weitere Aktivitäten wie Schreibworkshops (vgl. als Ergebnis eines solchen das Buch „Our homes, our stories“; van Ditzhuijzen, 2018), dass Care-Arbeiterinnen ihre Erfahrungen und Perspektiven auch vor einem größeren Publikum sichtbar machen.

All diese Instrumente und Praktiken schaffen also Sichtbarkeit sowohl innerhalb des Netzwerks der CareArbeiterinnen als auch darüber hinaus. Rother leitet aus Praktiken der Sichtbarkeit einen Statusgewinn ab, der die Care-Arbeiterinnen zu einer spezifischen politischen Klasse werden lässt (Rother, 2017:970). Demnach findet eine Identitätsbildung und -stärkung über entsprechende Praktiken der 
Sichtbarkeit statt. Wie die Beispiele zeigen, können die Frauen an vielfältigen Orten und in diversen Netzwerken Sichtbarkeit erlangen und damit ihre Anerkennung und ontologische Sicherheit stärken.

\subsection{Aushandlung von Zugehörigkeit}

Auch Zugehörigkeit, indem ein Individuum sich als Teil einer Gemeinschaft erfährt, schafft soziale Anerkennung und stärkt ontologische Sicherheit. Zugehörigkeit lässt sich über zwei Ebenen beschreiben: zum einen über ein persönliches, intimes Gefühl des Zu-Hause-Seins (place-belonging) sowie über eine diskursive Ebene (politics of belonging), die eine sozialräumliche Verortung von Ein- und Ausschlüssen aushandelt (Antonsich, 2010). Zur Betrachtung der Herstellung von ontologischer Sicherheit über die Dimension der Zugehörigkeit wollen wir zunächst den Blick auf die Gastfamilie lenken, die innerhalb des Arbeitsverhältnisses besonders relevant für die Frauen ist. Inwiefern Care-Arbeiterinnen als Familienmitglieder angesehen werden, ist in der Literatur umstritten, denn die emotionale Involviertheit einer ,familiären Handlungslogik“ (Schilliger, 2014:243) macht die persönliche Abgrenzung innerhalb des Arbeitsverhältnisses und das Einfordern von Arbeitsrechten für Care-Arbeiterinnen zu einer großen Herausforderung (Folbre, 2001; Schilliger, 2014). In feministischen geographischen Debatten zur ontologischen (Un-)Sicherheit werden das häusliche Umfeld und die Familie als Ort der Geopolitiken herausgestellt (Botterill et al., 2020:1157). Dabei wird sich das Konzept der ontologischen (Un-)Sicherheit zunutze gemacht, um die psychosozialen Prozesse innerhalb von familiären Geopolitiken zu untersuchen (ebd.:1143). Diese Perspektive wollen wir erweitern, indem wir aufzeigen, wie - über die Dimension der Zugehörigkeit - Familie als ein ,domestic site of geopolitics“ (ebd.:1139) für die Herstellung von ontologischer (Un-)Sicherheit relevant wird.

Care-Arbeiterinnen und ihre Arbeitgeber:innen finden meist über eine Agentur zueinander. Hierbei handelt es sich für die Care-Arbeiterinnen oft um einen passiven Akt der $\mathrm{Zu}$ weisung: ,they give you to the future employer" (S37:247f.). Das Ankommen in einer neuen Familie ist durch unterschiedliche Herausforderungen gekennzeichnet: Die Frauen müssen sich eine neue Arbeitsumgebung mit spezifischen Anforderungen erschließen und sich mit neuen Haushaltsmitgliedern arrangieren. So beschreibt Emily beispielsweise, dass die Kinder einer neuen Gastfamilie sie anfangs nicht in ihrem Zuhause haben wollten:

"I said, I cannot take this because they talk bad. They say 'I don't want you, Miss Emily. Get away from me. I don't like you to be here.' Something like that, but I understand, because they don't have any helper in their place in UK. And they don't have other people in their place and they are always in the house with the grandmother." (S37:656f.)
Auf der diskursiven Ebene der ,politics of belonging“ (Antonsich, 2010:649) wurde ihre sozialräumliche Inklusion also zunächst nicht anerkannt. Als Quasi-Familienmitglied nehmen sich die Frauen wahr, wenn ihnen ein wertschätzender Umgang und Vertrauen entgegengebracht werden, insbesondere durch das Übertragen von Verantwortung. So erlebt Emily sich selbst mit der Zeit als Quasi-Familienmitglied in dem Haushalt, in dem sie zuvor von den Kindern so schmerzlich abgewiesen wurde:

"Yes. This is, uh, it's like my home - everything. Because they're - the thing is, they trust me. And they are, uh, confident on me, what I'm doing." (S37:671f.)

Das Gefühl des Zu-Hause-Seins unterstreicht das Phänomen des ,,place-belonging“ (Antonsich, 2010:646). Auch andere Care-Arbeiterinnen heben diejenigen Arbeitgeber:innen positiv hervor, bei denen sie sich als Quasi-Familienmitglied erlebt haben. Arbeitgeber:innen wiederum beschreiben, wie das Ausgliedern der Care-Verpflichtungen ihnen dazu verhelfe, mit ihren Kindern qualitativ hochwertige Zeit zu verbringen, was mit Dankbarkeit gegenüber ihren Angestellten verbunden sei. Im Umkehrschluss aber auch heißen kann, dass sie den Care-Arbeiterinnen diese Form der Verbundenheit mit ihren Kindern absprechen.
"So, in the end, the interaction that I got with my daughters is more meaningful than what they used to be." (S60:465f.)

Marcus Yu Lung Chiu (2018) untersucht die wechselseitige Aushandlung von fiktiv verwandtschaftlichen Beziehungen zwischen Care-Arbeiterinnen und ihren Arbeitgeber:innen in Singapur. Mit dem Begriff der familization beschreibt er eine neue Sorte von Familie und einen identitätsstiftenden Prozess, der emotional intensiv erfahren wird. Demnach bewerten Care-Arbeiterinnen, die sich als Teil der Familie ihrer Arbeitgeber:innen verstehen, ihre Stimmung signifikant positiver (Chiu, 2018:109). An erster Stelle seiner politischen Forderungen steht daher, das Arbeitsumfeld der Care-Arbeiterinnen so zu gestalten, dass sie als Familienmitglieder behandelt werden (Wang und Liu, 2018:133). Dieser Perspektive steht allerdings die berechtigte kritische Einschätzung in der Literatur gegenüber, wonach die emotionale Involviertheit der Care-Arbeiterinnen über entsprechende Formen der familiären Zugehörigkeit dazu führt, dass Arbeitsrechte ausgehebelt werden (Folbre, 2001; Schilliger, 2014).

Die Möglichkeit der Care-Arbeiterinnen, ihre Anstellung zu wechseln, ist von unterschiedlichen Faktoren abhängig, wie beispielsweise von der Agentur, über die sie nach Singapur gekommen sind. Generell ist es für die Frauen jedoch ausgesprochen schwierig, in den vorherrschenden Hierarchien Kritik an aktuellen Arbeitsbedingungen anzubringen, da ihr Aufenthalt an einen Arbeitsvertrag in Singapur geknüpft ist. Sich frei eine Anstellung zu suchen, in der die eigenen 
Ansprüche und Bedürfnisse Berücksichtigung finden, ist daher nicht einfach zu realisieren. Maria berichtet von einem prekären Anstellungsverhältnis:

\begin{abstract}
"So, it was like for 18 months, I didn't have enough food. I had, to take my bath at the Queenstown Swimming Complex every Sunday, because I couldn't use the bathroom during Sundays. So, after 18 months, I felt like it's too much and the wife was not really nice. But I wanted to keep my work as long as I can. But during that time, I really could not take it anymore, so I eventually answered back. She didn't like it.“(S05:124f.)
\end{abstract}

Mit einer Frist von zehn Tagen wurde Maria daraufhin entlassen und musste sich unter hohem Zeitdruck eine neue Arbeitsstelle suchen, was ihr durch das Netzwerke ihrer Kirche gelang.

Die Suche nach einem Gefühl der Zugehörigkeit richtet sich oft auf die Gastfamilie, aber auch auf andere Kontexte. Die eigene Familie am Herkunftsort spielt eine wichtige Rolle für Gefühle der familiären Zugehörigkeit, jedoch bleibt der lokale Kontext des Arbeitsumfeldes in Singapur relevant. Die Frauen thematisieren hinsichtlich der Schaffung ontologischer Sicherheit über Zugehörigkeit - gleichermaßen wie für die Sichtbarkeit - multiple Orte und Gemeinschaften. Somit kommen Formen der Gruppenzugehörigkeit zum Tragen, die auf Herkunft, Sprache und Religion beruhen. Hier ist insbesondere die Zugehörigkeit zur Gemeinschaft der CareArbeiterinnen relevant. Rabitah, die ihren Lebensmittelpunkt zurück nach Indonesien verlegen möchte, um ihrer Familienplanung den Vorzug gegenüber ihrer Einkommenssituation zu geben, erläutert bezüglich des Miteinanders in der Akademie der Care-Arbeiterinnen:

"We greet each other. We talk to each other: 'Where are you from? Which part of Indonesia?' That is the starting of the communication. And then we get closer. We get more comfortable. That's why I call it a second family. And for those, who have any problem, they will just share. [...] The communication, the sharing. Everything, it makes - you know it makes - it can change someone's feeling." (S07:1260f.)

Das persönliche, intime Gefühl des Zu-Hause-Seins lässt sich demnach nicht nur auf einen Ort beziehen, sondern auch auf einen sozialen Kontext, in dem eine Verbundenheit und ein tiefes Vertrauen in die Zugehörigkeit zu anderen und damit auch in die eigene Position in der Welt existiert.

Rother (2017:960) legt dar, dass ein Individuum in einer transnationalen Perspektive multiple Positionen und Klassenzugehörigkeiten auf sich vereinen kann:

"Within a transnational space established by migration, the relationality becomes more complex; position can thus be defined not only in relation to other members of the society one lives in, but also in relation to the position oneself might hold in a different location." (Ebd.)

Multiple Zugehörigkeiten sehen wir bei den Frauen, indem Zugehörigkeit über die Gastfamilie hinaus auch über andere Orte und Netzwerke relevant wird. Zugehörigkeit wird schließlich subjektiv wahrgenommen, verkörpert und über Praktiken an den spezifischen Orten und in Netzwerken erlebt. Für das Empfinden ontologischer Sicherheit ist demnach nicht nur eine Gewissheit über die Kontinuität des Selbst relevant, sondern auch darüber, als Teil einer Gemeinschaft anerkannt zu werden.

\section{Fazit}

Die Arbeitsumgebung von Care-Arbeiterinnen ist durch die Fusionierung von Arbeitsplatz und Wohnraum einzigartig. Sie stellt die Frauen vor eine besondere Herausforderung bezüglich ihrer subjektiven Positionierung und sozialen Anerkennung. Dabei stellt insbesondere die Untersuchung dieser besonderen räumlichen Konstellation und der damit verbundenen Konsequenzen für die Arbeitsbedingungen der Frauen ein Forschungsdesiderat dar, dessen Untersuchung Aufschluss über das soziale, psychologische und ökonomische Wohlergehen der Frauen verspricht (Fong und Yeoh, 2020:704). In unserer Studie in Singapur haben wir anhand von qualitativen Interviews mit der Methode der FotoElizitation untersucht, wie Care-Arbeiterinnen ihr psychosoziales Wohlergehen über ihre subjektiv verkörperte Positionierung innerhalb dieser Arbeitsumgebung herstellen. Dabei konnten wir zeigen, dass die Konzepte der ontologischen (Un-)Sicherheit (Giddens, 1990) und der sozialen Anerkennung (Honneth, 2003) ausgesprochen fruchtbar für eine räumliche Analyse des psychosozialen Wohlergehens sind. Vorangegangene Studien zum Wohlergehen migrantischer Care-Arbeiterinnen zeigen, wie die Frauen vor dem Hintergrund starker Ungleichheiten Strategien der Grenzziehung in Bezug auf die Familie der Arbeitgeber:innen relevant machen, um sich innerhalb der Arbeitsumgebung zu positionieren.

Wir erweitern diese Perspektiven und zeigen unter Rückgriff auf das Konzept der ontologischen (Un-)Sicherheit und der sozialen Anerkennung dezidiert, dass die Räumlichkeit der Arbeitsbedingungen im Allgemeinen und die sozialräumlichen Dimensionen der Sichtbarkeit und $\mathrm{Zu}$ gehörigkeit im Besonderen für die Positionierungen der Care-Arbeiterinnen hoch relevant sind. Gerade für CareArbeiterinnen - aufgrund der speziellen Einheit von Arbeitsund Wohnort - sind die räumlichen Komponenten ontologischer Ver(un)sicherung wesentlich. Die Aushandlung und Anerkennung des Selbst, der sozialen Identität und eines Gefühls von Sicherheit findet über eine Verortung in der Welt und dieser inhärenten sozialen Beziehungen statt. Sichtbarkeit und Zugehörigkeit sind bei der Herstellung der subjek- 
tiv empfundenen Gewissheit des Selbst besonders relevante Dimensionen - alltäglich verkörpert in multiplen Orten und Netzwerken. Über eine Vielzahl dieser räumlichen Bezüge sind die Arbeiterinnen in der Lage, ontologische Sicherheit aktiv herzustellen und somit ihr psychosoziales Wohlergehen zu stärken.

Bei der Positionierung kommt schließlich insbesondere die Maßstabsebene des Körpers (Hyndman, 2004:309) zum Tragen. Die individuellen Erfahrungen und Empfindungen sind subjektiv verkörpert und gleichzeitig in übergeordnete Machtstrukturen von gender, class und race eingebettet. Abschließend wollen wir daher den Blick zurück auf eine strukturelle Ebene lenken. Neben ihrer individuellen Handlungsmacht sind die Care-Arbeiterinnen weiterhin stark vulnerabel, sodass auch über strukturelle Veränderungen ihre Anerkennung gestärkt und Ungleichheit weiter abgebaut werden muss. Auf einer räumlichen Ebene bedeutet dies insbesondere die Notwendigkeit, migrantischen Care-Arbeiterinnen strukturell Zugang zu Orten und Gemeinschaften außerhalb ihrer Arbeitsräume zu ermöglichen. Ein Wohnmodell, in dem Care-Arbeiterinnen außerhalb der Arbeitskonstellation leben, wäre dafür ein Anfang - die Schaffung von Zugängen sollte jedoch deutlich darüber hinausgehen.

Datenverfügbarkeit. Aufgrund der getroffenen Datenschutzvereinbarungen sind die Daten, auf denen dieser Artikel beruht, nicht öffentlich zugänglich.

Autorenmitwirkung. Die beiden Autorinnen haben den Artikel gemeinsam konzipiert. Die Datenerhebung und -analyse wurde von JD durchgeführt. Die Interpretation der Ergebnisse ist gemeinsam erfolgt. Bei dem Verfassen des Artikels hat IH unterstützend mitgewirkt.

Interessenkonflikt. Die Autor*innen erklären, dass kein Interessenkonflikt besteht.

Haftungsausschluss. Copernicus Publications bleibt in Bezug auf gerichtliche Ansprüche in veröffentlichten Karten und institutionellen Zugehörigkeiten neutral.

Danksagung. Wir danken Carolin Genz und Lucas Pohl für viele intensive Diskussionen zum Argument, Carl-Jan Dihlmann für die Unterstützung bei der Auswertung des Interviewmaterials sowie den anonymen Gutachter*innen für ihre konstruktive Kritik.

Finanzierung. The research for this paper was funded by the DFG-funded collaborative research center "Re-Figuration of Spaces" (CRC 1265) with the grant no. 290045248.
Begutachtung. This paper was edited by Benedikt Korf and reviewed by two anonymous referees.

\section{Literatur}

Antonsich, M.: Searching for Belonging - An Analytical Framework, Geography Compass, 4, 644-659, https://doi.org/10.1111/j.1749-8198.2009.00317.x, 2010.

Aulenbacher, B., Lutz, H., und Schwiter, K.: Gute Sorge ohne gute Arbeit? Einleitung, in: Gute Sorge ohne gute Arbeit? Livein-Care in Deutschland, Österreich und der Schweiz, Herausgeber*innen: Aulenbacher, B., Lutz, H., und Schwiter, K., Beltz Juventa, Weinheim, 7-17, 2021.

Bondi, L.: Feeling insecure: a personal account in a psychoanalytic voice, Soc. Cult. Geogr., 15, 332-350, https://doi.org/10.1080/14649365.2013.864783, 2014.

Botterill, K., Hopkins, P., und Sanghera, G. S.: Young people's everyday securities: pre-emptive and pro-active strategies towards ontological security in Scotland, Soc. Cult. Geogr., 20, 465-484, https://doi.org/10.1080/14649365.2017.1346197, 2019.

Botterill, K., Hopkins, P., und Sanghera, G. S.: Familial geopolitics and ontological security: intergenerational relations, migration and minority youth (in)securities in Scotland, Geopolitics, 25, 1138-1163, https://doi.org/10.1080/14650045.2018.1512098, 2020.

Brighenti, A. M.: Visibility in social theory and social research, Palgrave Macmillan, Basingstoke, 2010.

Chiu, M. Y. L.: Familization of Indonesian Domestic Workers in Singapore, in: Gender, Care and Migration in East Asia, Herausgeber*innen: Ogawa, R., Chan, R. K., Oishi, A. S., und Wang, L.-R., Springer Singapore, Singapore, 97-116, https://doi.org/10.1007/978-981-10-7025-9_5, 2018.

Chung, R. Y.-N., Liao, T. F., und Fong, E.: Data Collection for Migrant Live-In Domestic Workers: A Three-Stage Cluster Sampling Method, Am. Behav. Sci., 64, 709-721, https://doi.org/10.1177/0002764220910223, 2020.

Constable, N.: Maid to order in Hong Kong: Stories of migrant workers, Cornell paperbacks, Cornell University Press, Ithaca, 1997.

Crain, M., Poster, W., und Cherry, M. (Hrsg.): Invisible Labor. Hidden Work in the Contemporary World, University of California Press, Oakland, 2016.

Dirksmeier, P.: Bildbegriffe und ihre Reichweite zur Analyse von Gesellschaft-Raum-Verhältnissen, in: Visuelle Geographien: Zur Produktion, Aneignung und Vermittlung von RaumBildern, Herausgeber*innen: Schlottmann, A. und Miggelbrink, J., transcript, Bielefeld, 195-208, 2015.

Dobrusskin, J., Helbrecht, I., Born, A. M., und Genz, C.: Bildgestützte Interviews in der Raumforschung: Potenziale der FotoElizitation, in: Methoden der qualitativen Raumforschung. Ein interdisziplinäres Handbuch, Herausgeber*innen: Heinrich, A. J., Marguin, S., Million, A., und Stollmann, J., transcript, Bielefeld, 207-221, 2021.

Folbre, N.: The Invisible Heart: Economics and Family Values, New Press, New York, 2001.

Fong, E. und Shibuya, K.: Migration Patterns in East and Southeast Asia: Causes and Consequences, Annu. Rev. Sociol., 46, 511531, https://doi.org/10.1146/annurev-soc-121919-054644, 2020. 
Fong, E. und Yeoh, B. S.: Migrant Domestic Workers: Disadvantaged Work, Social Support, and Collective Strategies in East Asia, Am. Behav. Sci., 64, 703-708, 2020.

Gadamer, H.-G.: Wahrheit und Methode. Grundzüge einer philosophischen Hermeneutik, Mohr, Tübingen, 1990.

Giddens, A.: The Consequences of Modernity, Polity Press, Cambridge, 1990.

Giddens, A.: Modernity and self-identity: Self and society in the late modern age, Polity Press, Cambridge, 1991.

Hall, B. J., Garabiles, M. R., und Latkin, C. A.: Work life, relationship, and policy determinants of health and well-being among $\mathrm{Fi}$ lipino domestic Workers in China: a qualitative study, BMC public health, 19, 229, https://doi.org/10.1186/s12889-019-6552-4, 2019.

Helbrecht, I., Dobrusskin, J., Genz, C., und Pohl, L.: Imaginationen der Globalisierung, in: Am Ende der Globalisierung, Herausgeber*innen: Löw, M., Sayma, V., Schwerer, J., und Wolf, H., transcript, Bielefeld, 307-336, 2021.

Honneth, A.: Unsichtbarkeit. Stationen einer Theorie der Intersubjektivität, SuhrkampVerlag, Frankfurt am Main, 2003.

Hopkins, P., Hörschelmann, K., Benwell, M. C., und Studemeyer, C.: Young people's everyday landscapes of security and insecurity, Soc. Cult. Geogr., 20, 435-444, https://doi.org/10.1080/14649365.2018.1460863, 2019.

Hörschelmann, K. und Reich, E.: Entangled (In)Securities: Sketching the Scope of Geosocial Approaches for Understanding "Webs of (In)Security", Geopolitics, 22, 73-90, https://doi.org/10.1080/14650045.2016.1214821, 2017.

Huang, S. und Yeoh, B. S. A.: Emotional Labour and Transnational Domestic Work: The Moving Geographies of "Maid Abuse" in Singapore, Mobilities, 2, 195-217, https://doi.org/10.1080/17450100701381557, 2007.

Hyndman, J.: Mind the gap: bridging feminist and political geography through geopolitics, Plit. Geogr., 23, 307-322, https://doi.org/10.1016/j.polgeo.2003.12.014, 2004.

ILO - International Labour Organization: Global estimates of migrant workers and migrant domestic workers: results and methodology: Special focus on migrant domestic workers, ILO, Geneva, 2015.

ILO - International Labour Organization: Public attitudes towards migrant workers in Japan, Malaysia, Singapore, and Thailand, 2019.

Lai, Y. und Fong, E.: Work-Related Aggression in Home-Based Working Environment: Experiences of Migrant Domestic Workers in Hong Kong, Am. Behav. Sci., 64, 722-739, https://doi.org/10.1177/0002764220910227, 2020.

Lan, P.-C.: Maid Or Madam? Filipina Migrant Workers and the Continuity of Domestic Labor, Gender Soc., 17, 187-208, https://doi.org/10.1177/0891243202250730, 2003a.

Lan, P.-C.: Negotiating Social Boundaries and Private Zones: The Micropolitics of Employing Migrant Domestic Workers, Soc. Probl., 50, 525-549, https://doi.org/10.1525/sp.2003.50.4.525, 2003 b.

Marti, G.: The effects of multilevel governance on the rights of migrant domestic workers in Singapore, J. Ethn. Migr. Stud., 45, 1345-1360, https://doi.org/10.1080/1369183X.2018.1441614, 2019.

Massaro, V. A. und Williams, J.: Feminist Geopolitics, Geography Compass, 7, 567-577, https://doi.org/10.1111/gec3.12054, 2013.
Mele, C.: Spatial order through the family: the regulation of urban space in Singapore, Urban Geogr., 38, 1084-1108, https://doi.org/10.1080/02723638.2016.1187372, 2017.

MOM - Singaporean Ministry of Manpower: Foreign Domestic Worker Study 2015: Report on Findings, Ministry of Manpower Singapore, 2016.

MOM - Singaporean Ministry of Manpower: Foreign domestic worker eligibility, Ministry of Manpower Singapore, online aufrufbar: https://www.mom.gov.sg/ passes-and-permits/work-permit-for-foreign-domestic-worker/ eligibility-and-requirements/fdw-eligibility, letzter Zugriff: 15 January 2021a.

MOM - Singaporean Ministry of Manpower: Foreign workforce numbers, Ministry of Manpower Singapore, online aufrufbar: https://www.mom.gov.sg/documents-and-publications/ foreign-workforce-numbers, letzter Zugriff: 8 January $2021 \mathrm{~b}$.

Ogawa, R., Chan, R. K., Oishi, A. S., und Wang, L.-R. (Hrsg.): Gender, Care and Migration in East Asia, Series in Asian Labor and Welfare Policies, Springer Singapore, Singapore, OnlineRessourcen, 2018.

Parreñas, R. S., Kantachote, K., und Silvey, R.: Soft violence: migrant domestic worker precarity and the management of unfree labour in Singapore, J. Ethn. Migr. Stud., online first, https://doi.org/10.1080/1369183X.2020.1732614, 2020.

Pohl, L., Genz, C., Helbrecht, I., und Dobrusskin, J.: Need for shelter, demand for housing, desire for home: a psychoanalytic reading of home-making in Vancouver, Housing Studies, online first, https://doi.org/10.1080/02673037.2020.1857708, 2020.

Pratt, G. und Johnston, C.: Filipina Domestic Workers, Violent Insecurity, Testimonial Theatre and Transnational Ambivalence, Area, 46, 358-360, https://doi.org/10.1111/area.12138_8, 2014.

Rother, S.: Indonesian migrant domestic workers in transnational political spaces: agency, gender roles and social class formation, J. Ethn. Migr. Stud., 43, 956-973, https://doi.org/10.1080/1369183X.2016.1274567, 2017.

Schilliger, S.: Pflege ohne Grenzen? Polnische Pendelmigrantinnen in der 24h-Betreuung. Eine Ethnographie des Privathaushalts als globalisiertem Arbeitsplatz, Dissertation, Universität Basel, 2014.

Sekeráková Búriková, Z.: Invisible in homes, visible in cities: visibility and dis/empowerment in paid domestic work in London, Gender Place Cult., online first, https://doi.org/10.1080/0966369X.2020.1835832, 2020.

Summerfield, G., Pyle, J. L., und Desai, M.: Preface to the symposium: Globalizations, transnational migration, and gendered care work, Globalizations, 3, 281-282, https://doi.org/10.1080/14747730600869912, 2006.

van Bortel, T., Martin, S., Anjara, S., und Nellums, L. B.: Perceived stressors and coping mechanisms of female migrant domestic workers in Singapore, PloS one, 14, e0210717, https://doi.org/10.1371/journal.pone.0210717, 2019.

van Ditzhuijzen, K. (Hrsg.): Our homes, our stories: Voices of migrant domestic workers in Singapore, First edition, Humanitarian Organization for Migration Economics, Singapore, 150 S., 2018.

Vaquera, E., Aranda, E., und Sousa-Rodriguez, I.: Emotional Challenges of Undocumented Young Adults: Ontological Security, Emotional Capital, and Well-being, Soc. Probl., 64, 298-314, https://doi.org/10.1093/socpro/spx010, 2017. 
Wang, L.-R. und Liu, M. P. H.: Investigating the Well-Being of Migrant Care Workers in Taiwan from the Perspective of Social Inclusion, in: Gender, Care and Migration in East Asia, Herausgeber*innen: Ogawa, R., Chan, R. K., Oishi, A. S., und Wang, L.-R., Springer Singapore, Singapore, 117-136, https://doi.org/10.1007/978-981-10-7025-9_6, 2018.

Wessels, A.: Home sweet home? Work, life and well-being of foreign domestic workers in Singapore. Research report, Humanitarian Organization for Migration Economics, Singapore, 2015.

Yeoh, B. S. A. und Huang, S.: Transnational Domestic Workers and the Negotiation of Mobility and Work Practices in Singapore's Home-Spaces, Mobilities, 5, 219-236, https://doi.org/10.1080/17450101003665036, 2010.
Yeoh, B. S. A., Huang, S., und Lam, T.: Transnationalizing the 'Asian' family: imaginaries, intimacies and strategic intents, Global Netw., 5, 307-315, https://doi.org/10.1111/j.14710374.2005.00121.x, 2005.

Yu, T. W.: Foreign domestic workers' living conditions survey, Transient Workers Count Too, online aufrufbar: http://twc2.org. sg/wp-content/uploads/2016/07/FDW-Report_Final.pdf (letzter Zugriff: 15 January 2021), 2016. 